\title{
Numerical Solution for Pseudomonotone Variational Inequality Problems by Extragradient Methods*
}

\author{
Federica Tinti
}

September 2003

\begin{abstract}
In this work we analyze from the numerical viewpoint the class of projection methods for solving pseudomonotone variational inequality problems. We focus on some specific extragradient-type methods that do not require differentiability of the operator and we address particular attention to the steplength choice. Subsequently, we analyze the hyperplane projection methods in which we construct an appropriate hyperplane which strictly separates the current iterate from the solutions of the problem. Finally, in order to illustrate the effectiveness of the proposed methods, we report the results of a numerical experimentation.
\end{abstract}

\section{Introduction}

We consider the classical variational inequality problem $\operatorname{VIP}(\mathrm{F}, \mathrm{C})$, which is to find a point $x^{*}$ such that

$$
x^{*} \in C \quad<F\left(x^{*}\right), x-x^{*}>\geq 0 \quad \forall x \in C,
$$

where $C$ is a nonempty closed convex subset of $\left.\Re^{n},<\cdot, \cdot\right\rangle$ the usual inner product in $\Re^{n}$ and $F: \Re^{n} \rightarrow \Re^{n}$ is a continuous function. Let $C^{*}$ be the set of the solutions.

${ }^{*}$ Italian FIRB Project, Grant n. RBAU01JYPN 
In the special case where $C=\Re_{+}^{n}$, the problem (1) is a nonlinear complementary problem (NCP):

$$
x^{*} \geq 0, \quad F\left(x^{*}\right) \geq 0 \quad \text { and } \quad<x^{*}, F\left(x^{*}\right)>=0 .
$$

If $F$ is affine, $F(x)=M x+q$ where $M \in \Re^{n \times n}$ and $q \in \Re^{n}$, then the problem (1) is an affine variational inequality problem and (2) is a linear complementary problem (LCP).

Many methods have been proposed to solve VIP(F,C). The simplest of these is the projection method, which, starting from any $x^{0} \in C$, iteratively updates $x$ according to the formula

$$
x^{k+1}=P_{C}\left(x^{k}-\alpha F\left(x^{k}\right)\right),
$$

where $P_{C}($.$) denotes the orthogonal projection map onto C$ and $\alpha$ is a judiciously chosen positive steplength. Here, $P_{C}\left(x^{k}-\alpha F\left(x^{k}\right)\right)$ is the solution of the following quadratic programming problem

$$
\min _{x \in C} \frac{1}{2} x^{T} x-\left(x^{k}-\alpha F\left(x^{k}\right)\right)^{T} x .
$$

The projection method is based on the observation that $x^{*} \in C$ is a solution of (1) if and only if

$$
x^{*}=P_{C}\left(x^{*}-\alpha F\left(x^{*}\right)\right) .
$$

This method is very simple; indeed it uses only function evaluations and projections onto $C$, then it is easy to implement, uses little storage, and can readily exploit any sparsity or separable structure in $F$ or in $C$. Furthermore, the projection is easy to be obtained where $C$ is defined by linear and/or box constraints. However, the projection methods require restrictive assumption on $F$ for the convergence. The convergence analysis for the projection methods is based on the contractive properties of the operator $x \rightarrow x-\alpha F(x)$ :

if $F$ is strongly monotone (with constant $l$ ), i.e.

$\exists l>0 \quad$ s.t. $\quad<F(x)-F(y),(x-y)>\geq l\|x-y\|^{2} \quad \forall x, y \in C \quad x \neq y$, and $F(x)$ Lipschitz continuous on $C$ (with Lipschitz constant $L$ ), i.e.

$$
\exists L>0 \quad \text { s.t. } \quad\|F(x)-F(y)\| \leq L\|x-y\| \quad \forall x, y \in C,
$$


and if $\alpha \in\left(0,2 l / L^{2}\right)$, the projection method determines a succession $\left\{x^{k}\right\}$ convergent to a solution of (1) (see page 24 [15], [16]).

Marcotte and $\mathrm{Wu}[11]$ have shown that the projection algorithm converges for cocoercive variational inequalities. We recall that the mapping $F$ is cocoercive on $C$ if there exist a positive constant $\tilde{l}$ such that

$$
<F(y)-F(x), y-x>\geq \tilde{l}\|F(y)-F(x)\|^{2} \quad \forall x, y \in C .
$$

Any strongly monotone (with constant $l$ ) and Lipschitz continuous mapping (with Lipschitz constant $L$ ) is cocoecive with the constant $\tilde{l}=\frac{l}{L^{2}}$.

Furthermore, any cocoercive mapping is monotone, that is $<F(x)-F(y), x-$ $y>\geq 0 \quad \forall x, y \in C$, and Lipschitz continuous $\left(L=\frac{1}{l}\right)$, but the converse in not true. If $C^{*} \neq \emptyset$ and $\alpha \in(0,2 \tilde{l})$, the cocoercivity of the operator $F$ is sufficient to assure the convergence of the projection algorithm.

To relax the strong hypotheses required by the projection method enlarging the class of the problems that we can solve, the extragradient method was proposed; because of (3), $x^{*} \in C$ is a solution of (1) if and only if

$$
x^{*}=P_{C}\left(x^{*}-\alpha F\left(P_{C}\left(x^{*}-\alpha F\left(x^{*}\right)\right)\right)\right) ;
$$

then the basic idea of this method is to update $x$ according to the double projection formula

$$
x^{k+1}=P_{C}\left(x^{k}-\alpha F\left(P_{C}\left(x^{k}-\alpha F\left(x^{k}\right)\right)\right)\right) .
$$

The extragradient method was proposed in the first time by Korpelevich [9] as follows. Given $x^{0} \in C$, we generate a succession $\left\{x^{k}\right\}$ such that

$$
\bar{x}^{k}=P_{C}\left(x^{k}-\alpha F\left(x^{k}\right)\right) \quad x^{k+1}=P_{C}\left(x^{k}-\alpha F\left(\bar{x}^{k}\right)\right) .
$$

where $\alpha$ is constant for all iterations. In [1] and [19] the convergence of the extragradient method is proved under the following hypothesis: $C^{*} \neq \emptyset, F$ is a monotone and Lipschitz continuous mapping and $\alpha \in(0,1 / L)$ where $L$ is the Lipschitz constant.

A drawback is the choice of $\alpha$ when $L$ is unknown. Indeed, if $\alpha$ is too small, the convergence is slow; when $\alpha$ is too large, there might be no convergence at all. This remark is confirmed by the numerical results shown in Table 
1 where we report the number of iteration (iter), the number of function evaluations (nf), and the number of projections (np) for different choice of $\alpha$ when the extragradient method is applied on some test problems. The test problems are described in Table 3 of the Section 3.

Table 1: Analysis of the convergence of the extragradient method (4) for different values of $\alpha$.

\begin{tabular}{ccc}
\hline$\alpha$ & $\mathrm{np} / \mathrm{nf}$ & iter \\
\hline \multicolumn{3}{c}{ Kojima Shindo } \\
\hline $10^{-2}$ & $442 / 442$ & 221 \\
$10^{-1}$ & $76 / 76$ & 38 \\
1 & $-/-$ & - \\
\hline \multicolumn{3}{c}{ User OPT } \\
\hline $10^{-3}$ & $1326 / 1326$ & 663 \\
$10^{-2}$ & $184 / 184$ & 92 \\
$10^{-1}$ & - & - \\
\hline \multicolumn{3}{c}{ Braess Net } \\
\hline $10^{-2}$ & $472 / 472$ & 236 \\
$10^{-1}$ & $80 / 80$ & 40 \\
1 & $-/-$ & - \\
\hline
\end{tabular}

Then, Khobotov in [8] introduces the idea to perform an adaptive choice of $\alpha$, changing its value at each iteration as described in Section 2. If $C^{*} \neq \emptyset$, $F(x)$ is a monotone mapping and $\alpha$ choice suitable (see Section 2), then, the convergence of the scheme is proved.

The hypothesis on the Lipschitz continuity of $F$ is removed and an automatic (algorithmic) rule is devised to make easy a convenient choice of the steplength.

Furthermore, as we see in the following, we can generalize the results on the convergence of the scheme to pseudomonotone VIPs, enlarging the class of the problems that we can solve.

Consequently, the general scheme of the algorithm becomes:

$$
\bar{x}^{k}=P_{C}\left(x^{k}-\alpha_{k} F\left(x^{k}\right)\right) \quad x^{k+1}=P_{C}\left(x^{k}-\eta_{k} F\left(\bar{x}^{k}\right)\right),
$$


where $x^{0} \in C$ is the starting point. In addition to the scheme in [8], we have analyzed other variants of (5) (see [10], [7]), in which the values of $\alpha_{k}, \eta_{k}$ are found using backtracking schemes similar to that of the Armijo steplength rule. The aim of these variants is to accelerate the convergence.

In [8], [10], the choice of the steplength rules follows an adaptive rule but they assume that $\alpha_{k}=\eta_{k}$, while in [6] and [7], the extragradient method uses $\alpha_{k} \neq \eta_{k}$ with different backtracking procedures to determine the steplength $\alpha_{k}$. In the first case [6], one projection is required for any tentative step of the search, while in [7] only one evaluation of function is performed for any tentative step of the search. The last method is advantageous especially when the projection is computationally expensive.

Another class of the extragradient methods is the so called projection-contraction methods [17], where in the second projection a more general operator is used.

The idea of these algorithms is to choose a symmetric positive definite matrix $M \in R^{n \times n}$ and a starting point $x^{0} \in C$, and to iteratively update $x^{k}$, as follows:

$$
x^{k+1}=x^{k}-\gamma M^{-1}\left(T_{\alpha}\left(x^{k}\right)-T_{\alpha}\left(P_{C}\left(x^{k}-\alpha F\left(x^{k}\right)\right)\right),\right.
$$

where $\gamma \in \Re^{+}$and $T_{\alpha}=(I-\alpha F)$ in which $I$ is the identity matrix, $\alpha$ is chosen dynamically (in according to an Armijo type rule), so $T_{\alpha}$ is strongly monotone.

The geometric interpretation of the methods in [6] and [7] has been further on developed recently by Solodov in [18], devising an effective method. It consists of two steps per iteration: in the first step, an appropriate hyperplane is found which separates the current iterate from the solution of the problem; in the second step the next iterate is determined as the projection of the current iterate onto the intersection of the feasible set with the halfspace containing the solution set.

In all the algorithms with structure as in (5), (except that in [17], that requires the monotonicity of $F$ ), the convergence is stated under the assumptions that $C^{*} \neq \emptyset$ and the continuous mapping $F$ is pseudomonotone. This is shown in the theorems reported in Section 2 that generalize to pseu- 
domonotone case the results of the convergence obtained in [8], [6], [7]. See also [15] and [3].

It is not required $F$ to be Lipschitz continuous.

We recall that the mapping $F$ is pseudomonotone when the following condition holds

$$
<F(y), x-y>\geq 0 \rightarrow<F(x), x-y>\geq 0 \quad \forall x, y \in C .
$$

The paper is organized as follows.

In the Section 2 we give a survey of the above methods, pointing out its numerical features and we describe the different adaptive choices of $\alpha_{k}$.

To evaluate the effectiveness of the proposed methods, we have implemented them as M-script files of MatLab, downloadable at the URL

$$
\text { http://dm.unife.it/pn2o/software.html. }
$$

Since we assume that $C$ is defined by linear equalities and inequalities, in order to compute the projection $P_{C}(x)$, the quadratic program solver quadprog. $m$ is used (see the MatLab optimization toolbox [13]).

In the last section we report the numerical results obtained by running these codes on a set of test problems arising from the literature and collected at URL

http://dm.unife.it/pn2o/software.html.

\section{Numerical features of the class of extragradient methods}

\subsection{Khobotov's method}

In [8] Khobotov proves that if $F(x)$ is a continuous monotone function and $\alpha$ suitable choice of the steplength is performed, the extragradient method (4) is convergent to a solution of (1). The proof is interesting since it includes a discussion about the choice of $\alpha_{k}$.

We extended the Khobotov's theorem to a function $F(x)$ pseudomonotone. For completeness, we report the convergence theorem: 
Theorem 2.1. (see [8])

Let the set $C^{*}$ of solutions of (1) be non-empty, let $C$ be a closed convex set, $F(x)$ a continuous pseudomonotone operator in $x$. Then, from any initial point $x^{0} \in C$, if $\alpha_{k}$ is such that

$$
0<\alpha_{k} \leq \min \left\{\bar{\alpha}, \beta \frac{\left\|x^{k}-\bar{x}^{k}\right\|}{\left\|F\left(x^{k}\right)-F\left(\bar{x}^{k}\right)\right\|}\right\}
$$

with $\beta \in(0,1)$ and $\bar{\alpha}$ is equal to the maximum value of the step, then the extragradient method (4) is convergent to a solution $x^{*}$ of (1), i.e.,

$$
\lim _{k \rightarrow \infty} \min _{x^{*}}\left\|x^{*}-x^{k}\right\|_{2}=0 \quad x^{*} \in C^{*} .
$$

Proof. The proof of the theorem is based on the following condition

$$
\left\|x^{k+1}-x^{*}\right\|^{2} \leq\left\|x^{k}-x^{*}\right\|^{2}-\left\|x^{k}-\bar{x}^{k}\right\|^{2}+\alpha_{k}^{2}\left\|F\left(x^{k}\right)-F\left(\bar{x}^{k}\right)\right\|^{2} .
$$

We proof that this condition (9) holds under the pseudomonotonicity of the operator $F(x)$;

we see that, $\forall u, v \in C$,

$$
\begin{aligned}
\|u-v\|^{2} & =\left\|u-P_{C}(u)+P_{C}(u)-v\right\|^{2} \\
& =\left\|u-P_{C}(u)\right\|^{2}+\left\|v-P_{C}(u)\right\|^{2}-2<u-P_{C}(u), v-P_{C}(u)>;
\end{aligned}
$$

by the properties of the projection onto the convex set $C$

$$
<u-P_{C}(u), v-P_{C}(u)>\leq 0 \quad \forall v \in C ; \forall u \in \Re^{n},
$$

we obtain:

$$
\|u-v\|^{2} \geq\left\|u-P_{C}(u)\right\|^{2}+\left\|v-P_{C}(u)\right\|^{2} .
$$

Taking $v=x^{*}, u=x^{k}-\alpha_{k} F\left(\bar{x}^{k}\right)$, (with $x^{k+1}=P_{C}\left(x^{k}-\alpha F\left(\bar{x}^{k}\right)\right)$ ), we have

$$
\left\|x^{k}-\alpha_{k} F\left(\bar{x}^{k}\right)-x^{*}\right\|^{2} \geq\left\|x^{k}-\alpha_{k} F\left(\bar{x}^{k}\right)-x^{k+1}\right\|^{2}+\left\|x^{*}-x^{k+1}\right\|^{2},
$$

which leads to the inequality

$$
\begin{aligned}
\left\|x^{k+1}-x^{*}\right\|^{2} \leq & \left\|x^{k}-\alpha_{k} F\left(\bar{x}^{k}\right)-x^{*}\right\|^{2}-\left\|x^{k}-\alpha_{k} F\left(\bar{x}^{k}\right)-x^{k+1}\right\|^{2} \\
= & \left\|x^{k}-x^{*}\right\|^{2}+\left\|\alpha_{k} F\left(\bar{x}^{k}\right)\right\|^{2}-2<\alpha_{k} F\left(\bar{x}^{k}\right), x^{k}-x^{*}>-\left\|x^{k}-x^{k+1}\right\|^{2}+ \\
& -\left\|\alpha_{k} F\left(\bar{x}^{k}\right)\right\|^{2}+2<\alpha_{k} F\left(\bar{x}^{k}\right), x^{k}-x^{k+1}> \\
= & \left\|x^{k}-x^{*}\right\|^{2}-\left\|x^{k}-x^{k+1}\right\|^{2}+2<\alpha_{k} F\left(\bar{x}^{k}\right), x^{*}-x^{k+1}>
\end{aligned}
$$


Recalling that the operator $F(u)$ is pseudomonotone, since $x^{*} \in C^{*} \subset C$,

$$
<F\left(x^{*}\right), x-x^{*}>\geq 0 \rightarrow<F(x), x-x^{*}>\geq 0 \quad x \in C
$$

Consequently, if $x=\bar{x}^{k},<F\left(\bar{x}^{k}\right), x^{*}-\bar{x}^{k}>\leq 0$ and we have

$$
\begin{aligned}
<F\left(\bar{x}^{k}\right), x^{*}-x^{k+1}> & =<F\left(\bar{x}^{k}\right), x^{*}-\bar{x}^{k}>+<F\left(\bar{x}^{k}\right), \bar{x}^{k}-x^{k+1}> \\
& \leq<F\left(\bar{x}^{k}\right), \bar{x}^{k}-x^{k+1}>.
\end{aligned}
$$

Then we have from (11):

$$
\begin{aligned}
\left\|x^{k+1}-x^{*}\right\|^{2} \leq & \left\|x^{k}-x^{*}\right\|^{2}-\left\|x^{k}-x^{k+1}\right\|^{2}+2 \alpha_{k}<F\left(x^{k}\right), x^{*}-x^{k+1}> \\
\leq & \left\|x^{k}-x^{*}\right\|^{2}-\left\|x^{k}-x^{k+1}\right\|^{2}+2 \alpha_{k}<F\left(\bar{x}^{k}\right), \bar{x}^{k}-x^{k+1}> \\
\leq & \left\|x^{k}-x^{*}\right\|^{2}-\left\|x^{k}-\bar{x}^{k}\right\|^{2}-\left\|\bar{x}^{k}-x^{k+1}\right\|^{2}+ \\
& -2<x^{k}-\bar{x}^{k}, \bar{x}^{k}-x^{k+1}>+ \\
& +2 \alpha_{k}<F\left(\bar{x}^{k}\right), \bar{x}^{k}-x^{k+1}> \\
= & \left\|x^{k}-x^{*}\right\|^{2}-\left\|x^{k}-\bar{x}^{k}\right\|^{2}-\left\|\bar{x}^{k}-x^{k+1}\right\|^{2}+ \\
& +2<x^{k}-\alpha_{k} F\left(\bar{x}^{k}\right)-\bar{x}^{k}, x^{k+1}-\bar{x}^{k}> \\
\leq & \left\|x^{k}-x^{*}\right\|^{2}-\left\|x^{k}-\bar{x}^{k}\right\|^{2}-\left\|\bar{x}^{k}-x^{k+1}\right\|^{2}+ \\
& +2<x^{k}-\alpha_{k} F\left(x^{k}\right)-\bar{x}^{k}, x^{k+1}-\bar{x}^{k}>+ \\
& +2<\alpha_{k} F\left(x^{k}\right)-\alpha_{k} F\left(\bar{x}^{k}\right), x^{k+1}-\bar{x}^{k}>.
\end{aligned}
$$

Using (10), with $v=x^{k+1}, u=x^{k}-\alpha_{k} F\left(x^{k}\right)$, we obtain:

$$
<x^{k}-\alpha_{k} F\left(x^{k}\right)-\bar{x}^{k}, x^{k+1}-\bar{x}^{k}>\leq 0 .
$$

Then, it follows

$$
\left\|x^{k+1}-x^{*}\right\|^{2} \leq\left\|x^{k}-x^{*}\right\|^{2}-\left\|x^{k}-\bar{x}^{k}\right\|^{2}-\left\|\bar{x}^{k}-x^{k+1}\right\|^{2}+2 \alpha_{k}\left\|F\left(x^{k}\right)-F\left(\bar{x}^{k}\right)\right\|\left\|x^{k+1}-\bar{x}^{k}\right\| .
$$

For any $x^{k+1}, x^{k}, \bar{x}^{k}, \alpha_{k}$, we have:

$$
\left\|x^{k+1}-\bar{x}^{k}\right\|^{2}+\alpha_{k}^{2}\left\|F\left(x^{k}\right)-F\left(\bar{x}^{k}\right)\right\|^{2} \geq 2 \alpha_{k}\left\|F\left(x^{k}\right)-F\left(\bar{x}^{k}\right)\right\|\left\|x^{k+1}-\bar{x}^{k}\right\| ;
$$

then we obtain from (12):

$$
\left\|x^{k+1}-x^{*}\right\|^{2} \leq\left\|x^{k}-x^{*}\right\|^{2}-\left\|x^{k}-\bar{x}^{k}\right\|^{2}+\alpha_{k}^{2}\left\|F\left(x^{k}\right)-F\left(\bar{x}^{k}\right)\right\|^{2} .
$$

Furthermore, the proof runs as in [8]. 
In the proof of the Khobotov's theorem, at each $k$-th iteration it is possible to find a compact subset of $C, \widehat{C}_{k}$, where the function $F$ is Lipschitz continuous; we denote by $L_{k}$ the locally Lipschitz constant.

Since $\widehat{C}_{k} \supset \widehat{C}_{k+1} \supset \ldots$, it follows that

$$
L_{0} \geq L_{1} \geq \ldots \geq L_{k} \geq
$$

and it must $\alpha_{k} \in\left(0,1 / L_{k}\right)$.

Then, if $\left\{L_{k}\right\}$ are known, the succession $\left\{\alpha_{k}\right\}$ could be nondecreasing.

In the practice, estimates $\widetilde{L}_{k}$ for $L_{k}$ must be used; then for $\widetilde{L}_{k},(13)$ does not hold and $\alpha_{k}$ is found from the following rule

$$
0<\widehat{\alpha} \leq \alpha_{k} \leq \min \left\{\bar{\alpha}, \beta \frac{\left\|x^{k}-\bar{x}^{k}\right\|}{\left\|F\left(x^{k}\right)-F\left(\bar{x}^{k}\right)\right\|}\right\}
$$

where $\bar{\alpha}$ is the maximum value of the step, $0<\beta<1$ (usually $\beta \approx 0.8,0.9$ ) and $\widehat{\alpha}=\min \left(\bar{\alpha}, \beta / L_{0}\right)$.

From the proof of the theorem, we can state the following Algorithm (Algorithm choice- $\alpha$ ) for the choice of the steplength $\alpha_{k}$.

Algorithm choice- $\alpha$

a $\alpha=\alpha_{k-1}{ }^{*}($ initial step $)$

b compute $F\left(x^{k}\right)$

c compute $\bar{x}^{k}=P_{C}\left(x^{k}-\alpha F\left(x^{k}\right)\right)$ and $F\left(\bar{x}^{k}\right)$

If $F\left(\bar{x}^{k}\right)=0$ then $\bar{x}^{k} \in C^{*}$

else if

$$
\alpha>\beta \frac{\left\|x^{k}-\bar{x}^{k}\right\|}{\left\|F\left(x^{k}\right)-F\left(\bar{x}^{k}\right)\right\|}
$$

a reduction rule of $\alpha$ is applied

and go to (c)

else $\alpha_{k}=\alpha$, and

$$
x^{k+1}=P_{C}\left(x^{k}-\alpha F\left(\bar{x}^{k}\right)\right) .
$$

${ }^{*}$ At the initial iteration $\alpha=\bar{\alpha}$ 
We enumerate several techniques for the reduction of $\alpha_{k}$; the following reduction rule at the step (c) is suggested by Marcotte, in [10]:

$$
\alpha=\min \left\{\frac{\alpha}{2}, \frac{\left\|x^{k}-\bar{x}^{k}\right\|}{\sqrt{2}\left\|F\left(x^{k}\right)-F\left(\bar{x}^{k}\right)\right\|}\right\} .
$$

We note that this rule is not always effective: this arises when, at the initial iterations, $\alpha_{k}$ assumes a small value and, because of the initialization step $\alpha=\alpha_{k-1}$, this value does not change in all the next iterations. Figure (1) shows the behavior of the stepsize $\alpha_{k}$, as $k$ increases, when we use the reduction rule (15); this rule does not exploit the opportunity of an adaptive alteration of the initial value of $\alpha_{k}$.

A variant of Marcotte's algorithm consists in to modified the initialization rule at the step (a) of the Algorithm choice- $\alpha$ as follows:

$$
\alpha=\alpha_{k-1}+\left(\beta \frac{\left\|x^{k-1}-\bar{x}^{k-1}\right\|}{\left\|F\left(x^{k-1}\right)-F\left(\bar{x}^{k-1}\right)\right\|}-\alpha_{k-1}\right) \cdot \gamma,
$$

where $\gamma \in(0,1), \beta \in(0,1)$.

By this rule we enable the increase of the value of $\alpha$ with respect to $\alpha_{k-1}$. Then we devise the following reduction rule at the step (c)

$$
\alpha=\max \left\{\widehat{\alpha}, \min \left\{\xi \cdot \alpha, \beta \frac{\left\|x^{k}-\bar{x}^{k}\right\|}{\left\|F\left(x^{k}\right)-F\left(\bar{x}^{k}\right)\right\|}\right\}\right\},
$$

where $\xi \in(0,1)$.

Figure (2) shows the behavior of $\alpha_{k}$ for different test problems when the formulas (16), (17) are used, with $\beta=0.7, \xi=0.8, \gamma=0.9$.

We observe that in general, the number of iterations decreases, since the rules (16), (17) enable to exploit the possibility to use convenient values of $\alpha_{k}$ at any iteration.

Since $\alpha_{k}$ is an estimate of the inverse of the local Lipschitz constant we can substitute the Algorithm choice- $\alpha \alpha$ with the following rule

$$
\alpha_{k}=\beta \frac{\left\|x^{k}-\bar{x}^{k-1}\right\|}{\left\|F\left(x^{k}\right)-F\left(\bar{x}^{k-1}\right)\right\|},
$$

avoiding the loop of the algorithm. 
In this case, for the same test problem in Fig. 2, the behavior of $\alpha_{k}$ defined by (18), is similar to that observed for $\alpha_{k}$ stated by (16), (17) (see Fig. 3). Nevertheless, in this case the convergence is not assured. The sequence $x^{k}$ is convergent if $\alpha_{k}$ defined by (18) is such that

$$
\alpha_{k} \leq \bar{\beta} \frac{\left\|x^{k}-\bar{x}^{k}\right\|}{\left\|F\left(x^{k}\right)-F\left(\bar{x}^{k}\right)\right\|}
$$

where $\bar{\beta}>\beta$. This is not true in general, but in all the examined test problems the convergence is obtained.

\subsection{The Extragradient method with $\alpha_{k} \neq \eta_{k}$}

In [6], the author proposes the iterative scheme as in (5), where $\alpha_{k}>0$ is located through a bracketing search and $\eta_{k}=\frac{<F\left(\bar{x}^{k}\right), x^{k}-\bar{x}^{k}>}{\left\|F\left(\bar{x}^{k}\right)\right\|^{2}}$.

The idea behind the algorithm is the following.

Let $\partial H_{k}=\left\{x \in \Re^{n} \mid<F\left(\bar{x}^{k}\right), \bar{x}^{k}-x>=0\right\}$ be an hyperplane normal to $F\left(\bar{x}^{k}\right)$ passing through $\bar{x}^{k}$; all solutions $x^{*}$ of $\operatorname{VIP}(\mathrm{F}, \mathrm{C})$ lie on one side of $\partial H_{k}$; indeed for the pseudomonotonicity of $F$, for any $x^{*} \in C^{*}$, we have $<F\left(x^{*}\right), \bar{x}^{k}-x^{*}>\geq 0$ and, consequently, $<F\left(\bar{x}^{k}\right), \bar{x}^{k}-x^{*}>\geq 0$. If $x^{k}$ is on the other side, i.e. $<F\left(\bar{x}^{k}\right), \bar{x}^{k}-x^{k}><0$, then $\partial H_{k}$ separates $x^{k}$ from the solutions of $\operatorname{VIP}(\mathrm{F}, \mathrm{C})$ (see Prop. 6, [6]).

If $\eta_{k}=\frac{<F\left(\bar{x}^{k}\right), x^{k}-\bar{x}^{k}>}{\left\|F\left(\bar{x}^{k}\right)\right\|^{2}}, x^{k}-\eta_{k} F\left(\bar{x}^{k}\right)$ is the orthogonal projection of $x^{k}$ onto $\partial H_{k}$. Then $x^{k+1}$, obtained by the second equation of (4), is the orthogonal projection of $x^{k}$ onto this hyperplane $\partial H_{k}$ and onto $C$.

Iusem's algorithm requires three constants: $\epsilon \in(0,1)$ and $\widehat{\alpha}, \widetilde{\alpha}$ such that $\widetilde{\alpha} \geq \widehat{\alpha}>0$; the sequence $\alpha_{k}$ is computed so that $<F\left(\bar{x}^{k}\right), \bar{x}^{k}-x^{k}>\leq 0$, which is guaranteed to happen when $\alpha_{k} \in[\widehat{\alpha}, \widetilde{\alpha}]$.

Then the algorithm can be stated as follows [6]:

\section{Algorithm I}

a given $x^{0} \in C, k=0, r x=e^{\dagger}$;

b if $\|r x\|<T O L^{\ddagger}$ then stop else

\footnotetext{
${ }^{\dagger} e$ is a vector with entries equal to one.

${ }^{\ddagger} T O L$ is the final tolerance.
} 
chosen the initial value of the bracketing procedure $\widetilde{\alpha}_{k} \in[\widehat{\alpha}, \widetilde{\alpha}]$, where $\widetilde{\alpha}_{k}$ denote certain "candidate" of the steplength $\alpha_{k}$.

c compute $\widetilde{x}^{k}=P_{C}\left(x^{k}-\widetilde{\alpha}_{k} F\left(x^{k}\right)\right)$ and $F\left(\widetilde{x}^{k}\right)$

d If $F\left(\widetilde{x}^{k}\right)=0$ then $\widetilde{x}^{k} \in C^{*}$ stop

else (selection of $\alpha_{k}$ trough a finite bracketing procedure:)

if

$$
\left\|F\left(\widetilde{x}^{k}\right)-F\left(x^{k}\right)\right\| \leq \frac{\left\|\widetilde{x}^{k}-x^{k}\right\|^{2}}{2 \widetilde{\alpha}_{k}^{2}\left\|F\left(x^{k}\right)\right\|}
$$

then $\bar{x}^{k}=\widetilde{x}^{k}$

else find $\alpha_{k} \in\left(0, \widetilde{\alpha}_{k}\right)$, such that

$$
\begin{aligned}
& \epsilon \frac{\left\|\widetilde{x}^{k}-x^{k}\right\|^{2}}{2 \widetilde{\alpha}_{k}^{2}\left\|F\left(x^{k}\right)\right\|} \leq \| F\left(P_{C}\left(x^{k}-\alpha_{k} F\left(x^{k}\right)\right)-F\left(x^{k}\right) \| \leq \frac{\left\|\widetilde{x}^{k}-x^{k}\right\|^{2}}{2 \widetilde{\alpha}_{k}^{2}\left\|F\left(x^{k}\right)\right\|}(19)\right. \\
& \quad \bar{x}^{k}=P_{C}\left(x^{k}-\alpha_{k} F\left(x^{k}\right)\right) \\
& \quad \text { endif } \\
& \quad \text { if } F\left(\bar{x}^{k}\right)=0 \text { then } \bar{x}^{k} \in C^{*} \text { stop }
\end{aligned}
$$

else compute

$$
x^{k+1}=P_{C}\left(x^{k}-\frac{<F\left(\bar{x}^{k}\right), x^{k}-\bar{x}^{k}>}{\left\|F\left(\bar{x}^{k}\right)\right\|^{2}} F\left(\bar{x}^{k}\right)\right)
$$

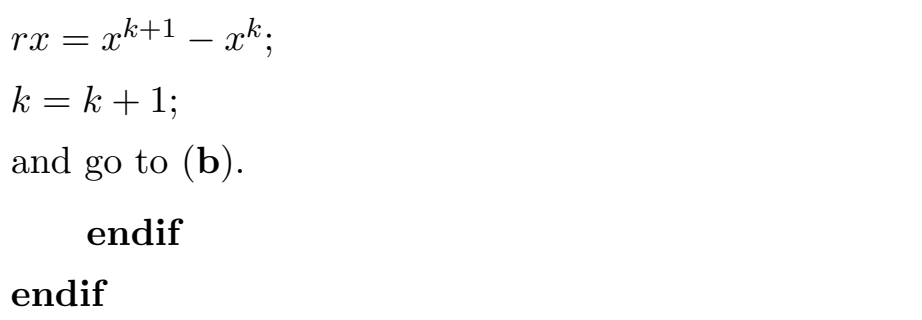

endif

endif

In the step (b) of the Iusem's algorithm, one possible rule to choose the initial value $\widetilde{\alpha}_{k}$ is

$$
\widetilde{\alpha}_{k}=\operatorname{median}\left(\widehat{\alpha}, \theta_{k}, \widetilde{\alpha}\right),
$$

where $\theta_{k}$ is suitably chosen.

In order to determine the stepsize $\alpha$ satisfying the required inequality (19), it 
is necessary to evaluate $P_{C}\left(x^{k}-\alpha F\left(x^{k}\right)\right)$ at any step of the search procedure. This means that the projections at the k-th iteration are those required for the bracketing search to determine $\alpha$, plus one more in the computation of $x^{k+1}$

In [6] (see Prop. 7), Iusem proves that if $C^{*} \neq 0$ and $F(x)$ is a continuous monotone function then this method is convergent to a solution of (1).

We extended the Prop. 7 to a function $F(x)$ continuous pseudomonotone, as follows:

Proposizione 2.2. (in [6])

Let the set $C^{*}$ of solutions of (1) be non-empty, let $C$ be a closed convex set, $F(x)$ a continuous pseudomonotone operator in $x$. Then, from any initial point $x^{0} \in C$, the sequence $\left\{x^{k}\right\}$ generated by Algorithm I is convergent to a solution of (1).

Proof. The proof of this proposition is based on the following condition

$$
\left\|x^{*}-x^{k+1}\right\|^{2} \leq\left\|x^{*}-x^{k}\right\|^{2}-\left\|P_{H_{k}}\left(x^{k}\right)-x^{k}\right\|^{2}-\left\|x^{k+1}-P_{H_{k}}\left(x^{k}\right)\right\|^{2},
$$

where $x^{*} \in C^{*}, H_{k}=\left\{x \in \Re^{n} \mid<F\left(\bar{x}^{k}\right), \bar{x}^{k}-x>\geq 0\right\}$.

We proof the condition (21) under the pseudomonotonicity of the operator $F(x)$.

From (7) with $x=\bar{x}^{k}, y=x^{*}$ we obtain

$$
<F\left(x^{*}\right), \bar{x}^{k}-x^{*}>\geq 0 \rightarrow<F\left(\bar{x}^{k}\right), \bar{x}^{k}-x^{*}>\geq 0 ;
$$

then $x^{*} \in C \cap H_{k}$, so $P_{C}\left(P_{H_{k}}\left(x^{*}\right)\right)=P_{H_{k}}\left(x^{*}\right)=x^{*}$.

Let $v^{k}=x^{k}-\eta_{k} F\left(\bar{x}^{k}\right)$ the orthogonal projection of $x^{k}$ onto the hyperplane $\partial H_{k}$, where $\partial H_{k}$ separes $x^{k}$ from the solution of $\operatorname{VIP}(\mathrm{F}, \mathrm{C})$; by Prop. 6 in [6], we obtain $x^{k} \notin H_{k}$, then $v_{k}=P_{H_{k}}\left(x^{k}\right)$.

It follows from (20) that $x^{k+1}=P_{C}\left(P_{H_{k}}\left(x^{k}\right)\right)$, then

$$
\left\|x^{*}-x^{k+1}\right\|^{2}=\left\|P_{C}\left(P_{H_{k}}\left(x^{*}\right)\right)-P_{C}\left(P_{H_{k}}\left(x^{k}\right)\right)\right\|^{2} .
$$

We apply the propriety of the projection onto the convex set $C$ (Prop. 2(ii) in $[6])$ :

$$
\left\|P_{C}(x)-P_{C}(y)\right\|^{2} \leq\|x-y\|^{2}-\left\|P_{C}(x)-x+y-P_{C}(y)\right\|^{2} \quad \forall x, y \in \Re^{n}
$$


first with $P_{C}($.$) and then with P_{H_{k}}($.$) as follows$

$$
\begin{aligned}
\left\|x^{*}-x^{k+1}\right\|^{2} \leq & \left\|P_{H_{k}}\left(x^{*}\right)-P_{H_{k}}\left(x^{k}\right)\right\|^{2}+ \\
& -\left\|P_{C}\left(P_{H_{k}}\left(x^{*}\right)\right)-P_{H_{k}}\left(x^{*}\right)+P_{H_{k}}\left(x^{k}\right)-P_{C}\left(P_{H_{k}}\left(x^{k}\right)\right)\right\|^{2} \\
\leq & \left\|x^{*}-x^{k}\right\|^{2}-\left\|P_{H_{k}}\left(x^{*}\right)-x^{*}+x^{k}-P_{H_{k}}\left(x^{k}\right)\right\|^{2}+ \\
& -\left\|P_{C}\left(P_{H_{k}}\left(x^{*}\right)\right)-P_{H_{k}}\left(x^{*}\right)+P_{H_{k}}\left(x^{k}\right)-P_{C}\left(P_{H_{k}}\left(x^{k}\right)\right)\right\|^{2} \\
\leq & \left\|x^{*}-x^{k}\right\|^{2}-\left\|P_{H_{k}}\left(x^{k}\right)-x^{k}\right\|^{2}-\left\|x^{k+1}-P_{H_{k}}\left(x^{k}\right)\right\|^{2} .
\end{aligned}
$$

Then, the proof runs as in Prop. 7 in [6].

In [7], Iusem and Svaiter present a method with the scheme similar to the previous algorithm but that requires just one projection onto $C$ for the computation of $\bar{x}^{k}$ and another one for $x^{k+1}$, i.e. only two projections per iteration, as in Korpelevich's method.

The algorithm requires the following parameters: $\epsilon \in(0,1)$ and $\widehat{\alpha}, \widetilde{\alpha}$ such that $\widetilde{\alpha} \geq \widehat{\alpha}>0$; the sequence $\alpha_{k}$ must be contained in $[\widehat{\alpha}, \widetilde{\alpha}]$; the scheme of the algorithm is:

Algorithm I-S

a given $x^{0} \in C, k=0, r x=e$;

b if $\|r x\|<T O L$ then stop

else

take an arbitrary stepsize $\alpha_{k} \in[\widehat{\alpha}, \widetilde{\alpha}]$,

c compute $z^{k}=x^{k}-\alpha_{k} F\left(x^{k}\right), v^{k}=P_{C}\left(z^{k}\right)$

d $\quad$ if $F\left(v^{k}\right)=0$ then $v^{k} \in C^{*}$ stop

e else

- compute

$$
\bar{j}=\min _{j \in Z^{+}}\left\{<F\left(2^{-j} P_{C}\left(z^{k}\right)+\left(1-2^{-j}\right) x^{k}\right), x^{k}-P_{C}\left(z^{k}\right)>\geq \frac{\epsilon}{\alpha_{k}}\left\|x^{k}-P_{C}\left(z^{k}\right)\right\|^{2}\right\}
$$

- compute $\beta_{k}=2^{-\bar{j}}$ 
- compute $y^{k}=\beta_{k} v_{k}+\left(1-\beta_{k}\right) x^{k}$

- compute $\eta_{k}=\frac{<F\left(y^{k}\right), x^{k}-y^{k}>}{\left\|F\left(y^{k}\right)\right\|^{2}}$

- compute the orthogonal projection of $x^{k}$ onto the hyperplane $\partial H_{k}$ :

$$
w^{k}=x^{k}-\eta_{k} F\left(y^{k}\right)
$$

- compute

$$
x^{k+1}=P_{C}\left(w^{k}\right)
$$

$r x=x^{k+1}-x^{k}$

$k=k+1$

then go to $(\mathbf{b})$.

endif

endif

In [7], Iusem and Svaiter observe that $\alpha_{k-1} \beta_{k-1}$ is an upper bound for the actual stepsize of the whole step from $x^{k-1}$ to $x^{k}$, and they suggest that $\alpha_{k-1}$, in the step (b), should be taken as

$$
\alpha_{k-1}=\operatorname{median}\left\{\widehat{\alpha}, \theta \beta_{k-1} \alpha_{k-1}, \widetilde{\alpha}\right\}
$$

where $\theta>1$ but not too large (for example $\theta=2$ ).

Note that along the search for the appropriate $\beta_{k}$, the right hand side of (23) is kept constant; then we evaluate $F$ at several points in the segment between $v^{k}$ and $x^{k}$, no orthogonal projection onto $C$ is required during the search, besides the computation of $v^{k}$ and $x^{k+1}$.

We observe that a too small value of $\epsilon$ might induce a loss of precision of the algorithm; on the other hand, a value of $\epsilon$ close to 1, make the inequality in (23) too tight, increasing the value of $j$, and therefore decreasing $\beta_{k}$, and lengthening the bracketing search. It follows that $\epsilon$ should not be close to either 0 or 1 .

In [7] (see Prop. 4), Iusem and Svaiter prove that if $C^{*} \neq 0$ and $F(x)$ is a continuous monotone function then this method is convergent to a solution of (1).

We extended the Prop. 4 to a function $F(x)$ continuous pseudomonotone, as follows: 
Proposizione 2.3. (in [7])

Let the set $C^{*}$ of solutions of (1) be non-empty, let $C$ be a closed convex set, $F(x)$ a continuous pseudomonotone operator in $x$. Then from any initial point $x^{0} \in C$, the sequence $\left\{x^{k}\right\}$ generated by Algorithm I-S is convergent to a solution of (1).

Proof. The proof of this proposition is based on the following condition

$$
\left\|x^{k+1}-x^{*}\right\|^{2} \leq\left\|x^{k}-x^{*}\right\|^{2}-\left\|w^{k}-x^{k}\right\|^{2}-\left\|P_{C}\left(w^{k}\right)-w^{k}\right\|^{2},
$$

where $x^{*} \in C^{*}$.

Let $L_{k}=\left\{x \in \Re^{n} \mid<F\left(y^{k}\right), x^{k}-y>\leq 0\right\}$; using the pseudomonotonicity of $F$,

$$
<F\left(x^{*}\right), y^{k}-x^{*}>\geq 0 \rightarrow<F\left(y^{k}\right), y^{k}-x^{*}>\geq 0,
$$

we obtain that $x^{*} \in L_{k}$; on the other hand, $P_{C}\left(x^{*}\right)=x^{*}$.

By Prop. 3(iii) in [7], $x^{k}$ does not belong to $L_{k}$; then using (24), it follows

$$
P_{L_{K}}\left(x^{k}\right)=P_{\partial H_{K}}\left(x^{k}\right)=w^{k}
$$

Then, from the propriety of the projection (22) and from (25) we obtain

$$
\begin{aligned}
\left\|x^{k+1}-x^{*}\right\|^{2} & =\left\|P_{C}\left(w^{k}\right)-P_{C}\left(x^{*}\right)\right\|^{2} \\
& \leq\left\|w^{k}-x^{*}\right\|^{2}-\left\|P_{C}\left(w^{k}\right)-w^{k}\right\|^{2} \\
& =\left\|P_{L_{K}}\left(x^{k}\right)-P_{L_{K}}\left(x^{*}\right)\right\|^{2}-\left\|P_{C}\left(w^{k}\right)-w^{k}\right\|^{2} \\
& \leq\left\|x^{k}-x^{*}\right\|^{2}-\left\|P_{L_{K}}\left(x^{k}\right)-x^{k}\right\|^{2}-\left\|P_{C}\left(w^{k}\right)-w^{k}\right\|^{2} .
\end{aligned}
$$

Then, the proof runs as in Prop. 4 in [7].

From the computational point of view this method appears not effective since the convergence is very slowly, then we do not report in Section 3 the numerical results of this method, because they were rather poor. Indeed, we observe that frequently the hyperplane $\partial H_{k}$ is near to the point $x^{k}$ and the next iteration $x^{k+1}=P_{C}\left(\bar{x}^{k}\right)$ is not much different from $x^{k}$ and the convergence of the algorithm is very slow.

The interest forward the methods in [6] and [7] is justified by the fact that they are based on the same idea of the method of Solodov and Svaiter, discussed later in 2.4 . 


\subsection{Solodov and Tseng (S-T) method}

In [17], Solodov and Tseng propose a new class of methods for solving variational inequality problem, called projection-contraction methods, where the second projection is a more general operator:

$$
\bar{x}^{k}=P_{C}\left(x^{k}-\alpha_{k} F\left(x^{k}\right)\right), \quad x^{k+1}=x^{k}-\gamma M^{-1}\left(T_{\alpha}\left(x^{k}\right)-T_{\alpha}\left(P_{C}\left(\bar{x}^{k}\right)\right),\right.
$$

where $\gamma \in \Re^{+}$and $T_{\alpha}=(I-\alpha F)$; here $I$ is the identity matrix, $\alpha$ is chosen dynamically (in according to an Armijo type rule), such that $T_{\alpha}$ is strongly monotone.

Unlike the classical extragradient method (5), these methods require only one projection per iteration, rather then two, and they have an additional parameter, the scaling matrix $M$, that can be chosen to accelerate the convergence.

$M$ must be a symmetric positive matrix.

The scheme of the method is the following.

\section{Algorithm S-T}

a choose $x^{0} \in \Re^{n}, \alpha_{-1}>0, \theta \in(0,2), \rho \in(0,1), \beta \in(0,1), M \in \Re^{n \times n}$

b $\bar{x}^{0}=0, k=0, r x=e$

c if $\|r x\|<T O L$ then stop

else

$$
\alpha=\alpha_{k-1}, \text { flag }=0
$$

d if $F\left(x^{k}\right)=0$ then $x^{k} \in C^{*}$ stop

else

while

$$
\begin{gathered}
\left(\alpha\left(x^{k}-\bar{x}^{k}\right)^{T}\left(F\left(x^{k}\right)-F\left(\bar{x}^{k}\right)\right)>(1-\rho)\left\|x^{k}-\bar{x}^{k}\right\|^{2}\right) \operatorname{or}(\text { flag }=0) \\
\text { if } \text { flag } \neq 0 \text { then } \alpha=\alpha_{k-1} \beta \text { endif } ; \\
\text { update } \bar{x}^{k}=P_{C}\left(x^{k}-\alpha F\left(x^{k}\right)\right), \text { compute } F\left(\bar{x}^{k}\right) \\
\text { flag }=\text { flag }+1
\end{gathered}
$$




\section{endwhile}

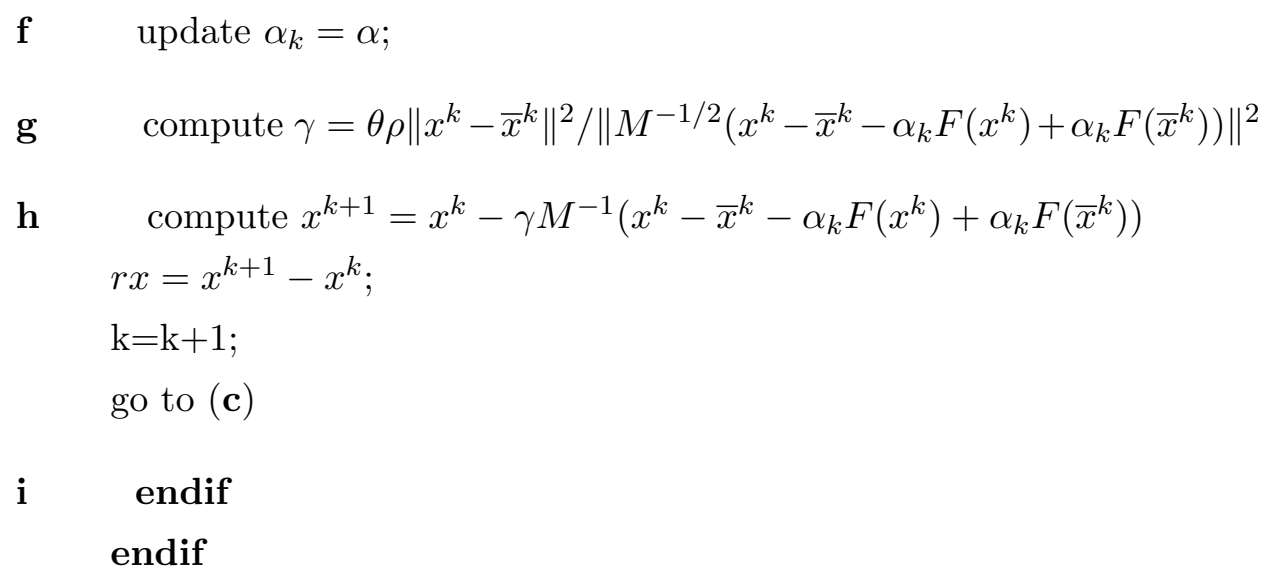

In this algorithm the condition (27) may be viewed as a local approximation to the condition $\alpha<1 / L_{k}$, where the local Lipschitz constant $L_{k}$ is given by

$$
L_{k}=\left(x^{k}-\bar{x}^{k}\right)^{T}\left(F\left(x^{k}\right)-F\left(\bar{x}^{k}\right)\right) /\left\|x^{k}-\bar{x}^{k}\right\|^{2} .
$$

Then (27) reduces to $\alpha \leq(1-\rho) / L_{k}$.

The convergence is proved under the assumption that a solution of (1) exists and that the operator $F$ is monotone.

The rule (27) requires one projection and one function evaluation for any step of the search procedure. Another function evaluation is required to complete any iteration.

In Table 2, we shown, for $\beta=0.3$ and $M=I$, the behavior of the method as $\theta$ and $\rho$ assumes different values. In general, the choice of these parameters significantly affects the effectiveness of the method. 


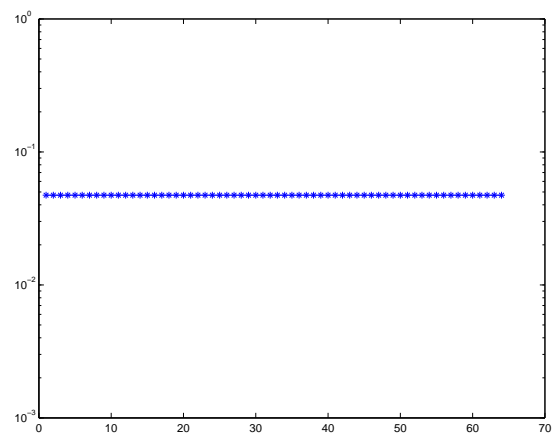

(a) Kojima Shindo $\alpha_{k}=0.0472 \forall k$

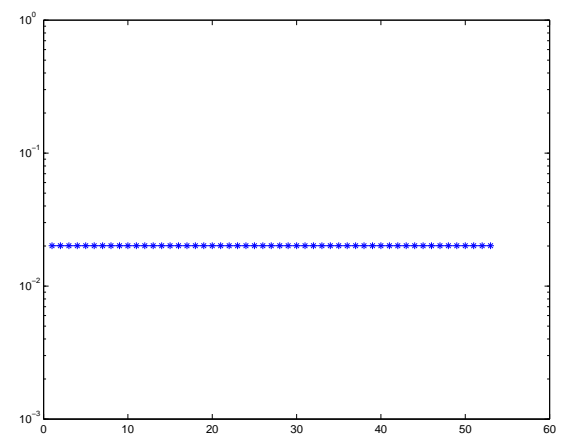

(b) User OPT $\alpha_{k}=0.0201 \forall k$

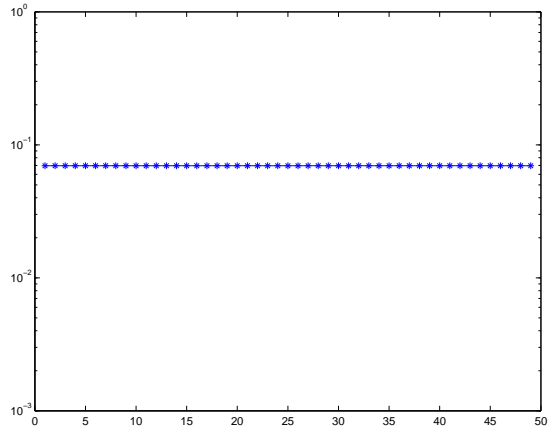

(c) Braess Network $\alpha_{k}=0.0697 \forall k$

Figure 1: Behavior of $\alpha_{k}$ with reduction rule (15). 


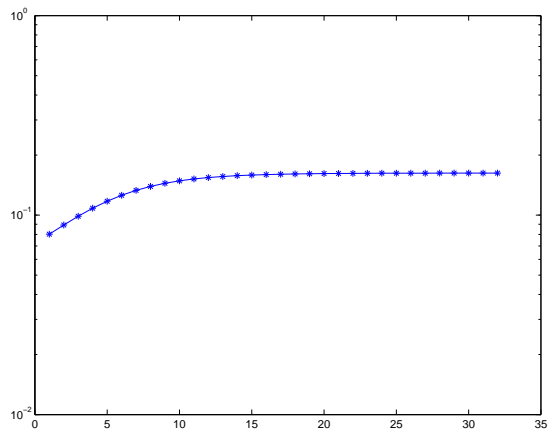

(a) Kojima Shindo

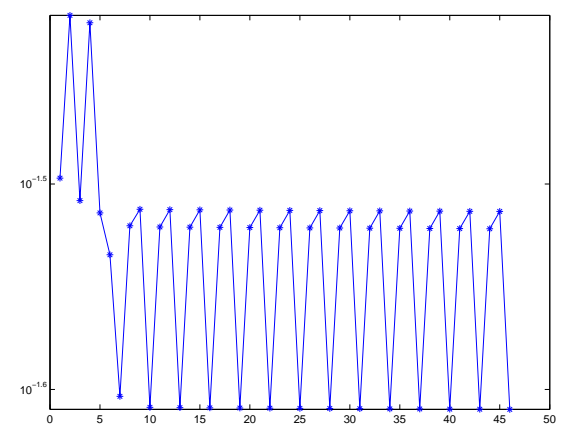

(b) User OPT

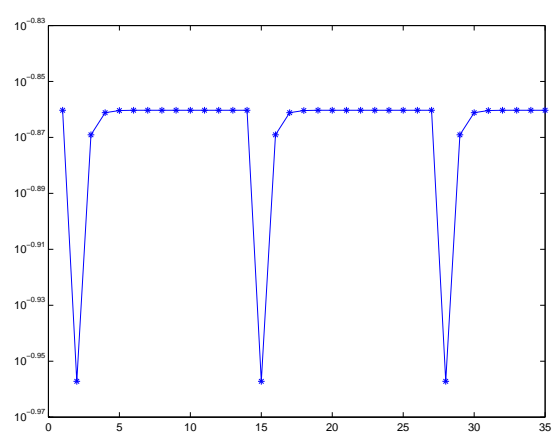

(c) Braess Network

Figure 2: Behavior of $\alpha_{k}$ with rules (16), (17); $\beta=0.7, \xi=0.8, \gamma=0.9$. 


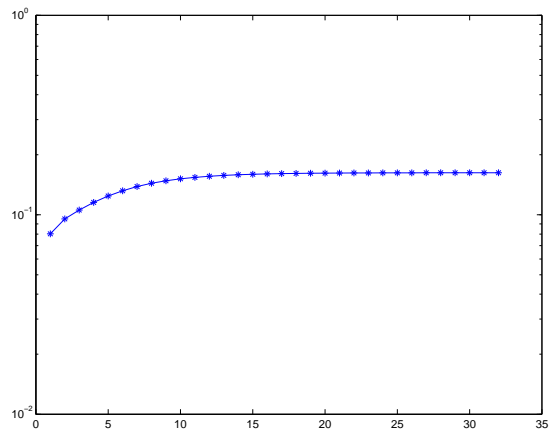

(a) Kojima Shindo

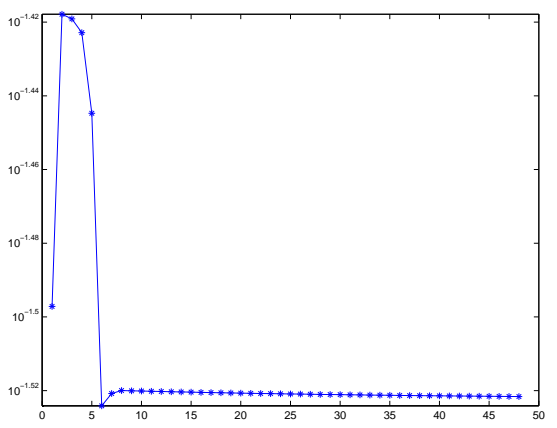

(b) User OPT

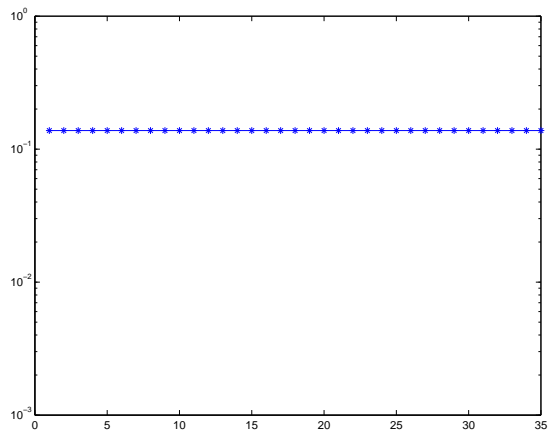

(c) Braess Network

Figure 3: Behavior of $\alpha_{k}$ with $\operatorname{rule}(18) ; \beta=0.7, \widetilde{\beta}=0.9$. 


\begin{tabular}{|c|c|c|c|c|}
\hline$\stackrel{120}{0}$ & $\underset{.}{ \pm}$ & 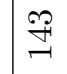 & $\stackrel{20}{2}$ & 先 \\
\hline $\begin{array}{c}0 \\
-1 \\
11 \\
0\end{array}$ & $\frac{\vec{\xi}}{\xi}$ & 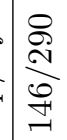 & 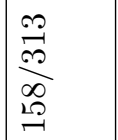 & 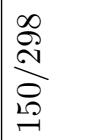 \\
\hline ت. & 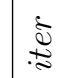 & $\stackrel{2}{\infty}$ & $\underset{-}{\stackrel{ }{O}}$ & $\stackrel{\leftrightarrow}{\leftrightarrow}$ \\
\hline$\underset{11}{11}$ & $\frac{\text { ह }}{\frac{\xi}{2}}$ & $\mid \begin{array}{l}\overrightarrow{\mid} \\
\overrightarrow{0} \\
-\infty \\
\infty \\
\infty \\
\infty\end{array}$ & $\underset{\stackrel{N}{N}}{\stackrel{N}{\hat{~}}}$ & $\begin{array}{l}\stackrel{\infty}{\infty} \\
\frac{2}{\not 2}\end{array}$ \\
\hline $\begin{array}{l}19 \\
0 \\
11\end{array}$ & $\underset{.}{\Phi}$ & $\mathscr{0}$ & $\stackrel{\infty}{\sim}$ & ठే \\
\hline 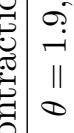 & $\frac{\frac{2}{\xi}}{\frac{\xi}{2}}$ & 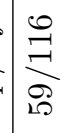 & $\stackrel{ }{\stackrel{ }{\vec{N}}}$ & 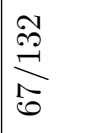 \\
\hline $\begin{array}{l}-1 \\
0 \\
11\end{array}$ & $\underset{ \pm}{\Phi}$ & $\stackrel{9}{\not}$ & กิ่ & $\mathscr{O}$ \\
\hline 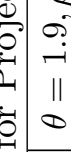 & $\frac{\vec{\varepsilon}}{\xi}$ & 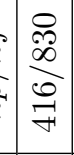 & $\frac{\infty}{\stackrel{0}{12}}$ & 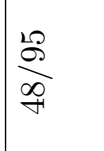 \\
\hline 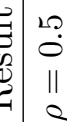 & 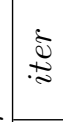 & $\vec{\infty}$ & $\infty$ & $\infty$ \\
\hline 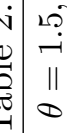 & $\frac{\vec{\varepsilon}}{\xi}$ & $=\begin{array}{l}\infty \\
\stackrel{-}{+} \\
\stackrel{\infty}{+}\end{array}$ & 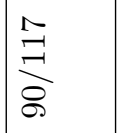 & 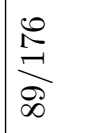 \\
\hline $\begin{array}{l}-1 \\
0 \\
11\end{array}$ & $\underset{\Xi}{\Xi}$ & 章 & 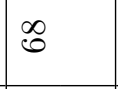 & 80 \\
\hline م. & $\frac{\grave{\xi}}{\xi}$ & 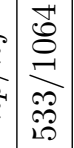 & $\underset{ }{\stackrel{ }{I}}$ & 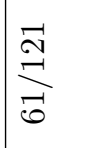 \\
\hline 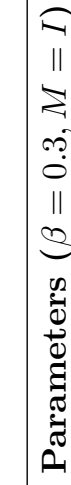 & & 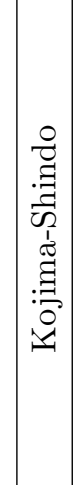 & 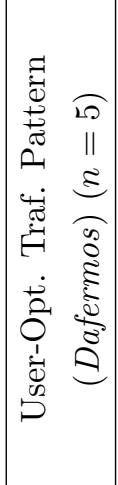 & 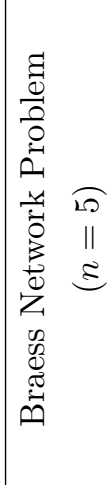 \\
\hline
\end{tabular}




\subsection{Solodov and Svaiter (S-S) method}

Finally, we have analyzed a projection algorithm that was proposed by Solodov and Svaiter, in [18].

This algorithm allows a geometric interpretation as in [6] and [7] (see Fig. 4):

let $x^{k}$ be the current approximation of the solution of $\operatorname{VIP}(\mathrm{F}, \mathrm{C})$; first, we compute the point $P_{C}\left(x^{k}-\mu_{k} F\left(x^{k}\right)\right)$; next, we search the line segment between $x^{k}$ and $P_{C}\left(x^{k}-\mu_{k} F\left(x^{k}\right)\right)$ for a point $z^{i}$ such that the hyperplane

$$
\partial H_{k}=\left\{x \in \Re^{n} \mid<F\left(z^{k}\right), x-z^{k}>=0\right\}
$$

strictly separes $x^{k}$ from the solution of the VIP(F,C) $x^{*}$.

To compute $z^{k}$, an Armijo-type procedure is used, i.e., $z^{k}=x^{k}-\eta_{k} r\left(x^{k}, \mu_{k}\right)$ where $\eta_{k}=\gamma^{\bar{i}} \mu_{k}$ with $\bar{i}$ being the smallest nonnegative integer $i$ satisfying

$$
\left.<F\left(x^{k}-\gamma^{i} \mu_{k} r\left(x^{k}, \mu_{k}\right)\right), r\left(x^{k}, \mu_{k}\right)>\geq \frac{\sigma}{\mu_{k}}\left\|r\left(x^{k}, \mu_{k}\right)\right\|^{2}\right\}
$$

and $r\left(x^{k}, \mu_{k}\right)=x^{k}-P_{C}\left(x^{k}-\mu_{k} F\left(x^{k}\right)\right)$ is the projected residual function; after the hyperplane $\partial H_{k}$ is constructed, the next iterate $x^{k+1}$ is computing by projecting $x^{k}$ onto the intersection between the feasible set $C$ with the halfspace $H_{k}=\left\{x \in \Re{ }^{n} \mid<F\left(z^{k}\right), x-z^{k}>\leq 0\right\}$ which contain the solution set $C^{*}$.

The scheme of the Solodov and Svaiter algorithm is reported in the following.

\section{Algorithm S-S}

a choose $x^{0} \in C, \eta_{-1}>0, \gamma \in(0,1), \sigma \in(0,1), \theta>1, k=0, r x=e$

b if $\|r x\|<T O L$ then stop

else

$$
\text { compute } \mu_{k}=\min \left\{\theta \eta_{k-1}, 1\right\}
$$

c if $r\left(x^{k}, \mu_{k}\right):=x^{k}-P_{C}\left(x^{k}-\mu_{k} F\left(x^{k}\right)\right)=0$ then $x^{k} \in C^{*}$ stop

d else compute

$$
\bar{i}=\min _{i \in Z^{+}}\left\{<F\left(x^{k}-\gamma^{i} \mu_{k} r\left(x^{k}, \mu_{k}\right)\right), r\left(x^{k}, \mu_{k}\right)>\geq \frac{\sigma}{\mu_{k}}\left\|r\left(x^{k}, \mu_{k}\right)\right\|^{2}\right\}
$$


where $\eta_{k}=\gamma^{\bar{i}} \mu_{k}$

e compute $z^{k}=x^{k}-\eta_{k} r\left(x^{k}, \mu_{k}\right)$

f compute the halfspace $H_{k}=\left\{x \in \Re^{n} \mid<F\left(z^{k}\right), x-z^{k}>\leq 0\right\}$

g $\quad$ compute $x^{k+1}=P_{C \cap H_{k}}\left(x^{k}\right)$

$r x=x^{k+1}-x^{k}$

$k=k+1$

go to $(\mathbf{b})$

h endif

endif

Also in this method are needed only two projection per iteration.

This method should be especially effective when feasible sets are "no simpler" than general polyhedra; in this case, adding one more linear constraint to perform a projection onto $C \cap H_{k}$ doesn't increase the cost compared to projecting onto the feasible set $C$.

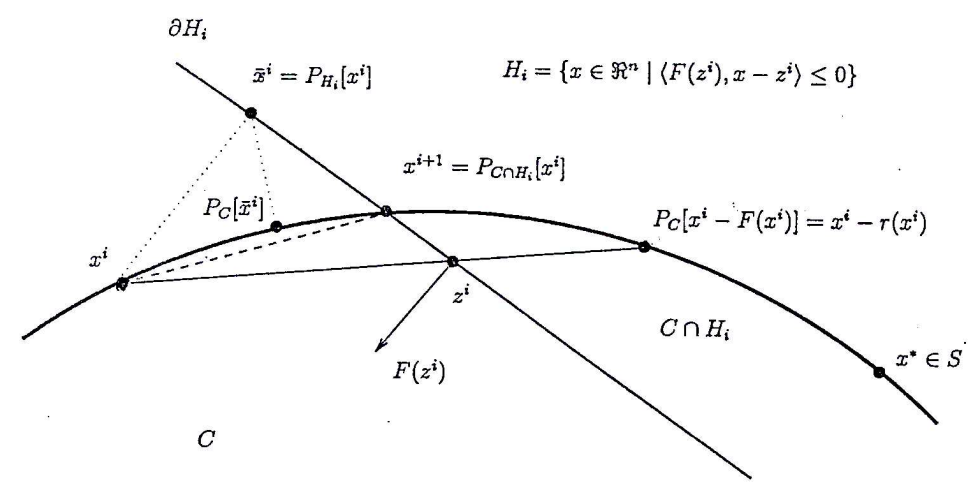

Figure 4: Comparison between Iusem Svaiter method [7] and Solodov and Svaiter method [18]

In Figure 4,we analyze the differences between the Iusem and Svaiter method in [7] and the Solodov's method. In [7], $x^{k}$ is projected first onto the separating hyperplane $\partial H_{k}$ and then onto $C$. If $x^{*}$ near $\partial H_{k}, P_{C}\left(\bar{x}^{k}\right)$ can computationally are equal to $x^{k}$ and the algorithm does not converge. 
In [18], the second projection step in our method is onto the intersection $C \cap H_{k}$. We can observe that the iterate $x^{k+1}$ is closer to the solution set $C^{*}$ than the iterate computed by the method in [7].

In [18] it is shown that this method is convergent to a solution of the variational inequality problem under the only assumption that $F$ is continuous and pseudomonotone. 


\section{Computational experience}

In order to evaluate the effectiveness of the extragradient methods discussed in the previous section, we consider a set of test problems arising from the literature (see the list in Table 3 ).

The M-function files implementing the considered test problems are downloadable at URL (http://dm.unife.it/pn2o/software.html).

We report in Table 4 the numerical results obtained by the MatLab M-script files implementing the considered methods. These codes can be downloadable at the URL (http://dm.unife.it/pn2o/software.html).

For the test problems with the suffix 'box' in the name of the input script files, the feasible region is given by the nonnegative orthant $x \in \Re_{+}^{n}$; they are NCPs. The other test problems are VIPs.

We choose very simple feasible regions so that the solver for inner quadratic programming problem has a low cost.

The starting point for all methods are feasible.

But, if we start from an unfeasible point, the first projection enables us to determine a feasible point that can be used as initial iterate.

All MatLab codes are run on a Notebook personal computer (ACER TravelMate 435LC, P-IV 3.06GHz) under MatLab version 6.5.0.180913a R13.

The following remarks can be drawn:

- between the three variants of the extragradient method, those related to (16)-(17) and (18) are more effective; the scheme related to (18) has near the same number of iterations with respect that related to (16)-(17) but the number of the projections and the number of the function evaluations are smaller; we remark the effectiveness of the extragradient method combined with (18) when we have to solve an NCP;

- the convergence of the S-T method is holds for monotone maps; the method has a better performance with respect the extragradient methods and it is very efficient for an affine VIP (see the test problem 
HPHard); for several test problems the number of iterations of this method appears convenient with respect to the S-S method; nevertheless the execution time of the S-S method can be smaller than that of the S-T method; but half of the projections of the S-S method has a different feasible region and then the number of projections are not comparable. Furthermore, the behavior of the S-T method strongly depends on the choices of its parameters (see Table 2).

For monotone VIPs, we can be find convenient parameters so that the method is competitive with the others.

- For pseudomonotone VIPs, the S-S method appears in general very effective (only for the test problem HpHard the behavior of the S-S method is poor); indeed, the numbers of iterations of the S-S method is less than those of all the other methods (except for the S-T method, however, that requires the monotonicity of $F$ ); but the complexity of each iteration can be larger of that of the other methods. Indeed the number of function evaluations can be greater than those of the extragradient method combined with the rule method (18) or (16) (17) and half of the projections has a different computational complexity since the feasible region is complicated by an additional (linear) constraint. Then the effectiveness of the S-S method can depend on the structure of the feasible region, on the performance of the solver for the inner quadratic programming problem and on the analytical form of the mapping $F$.

We remark, in particular, the loss of the efficient for the NCPs, where the feasible region given by the nonnegative orthant significantly changes by the addition of a linear inequality. 


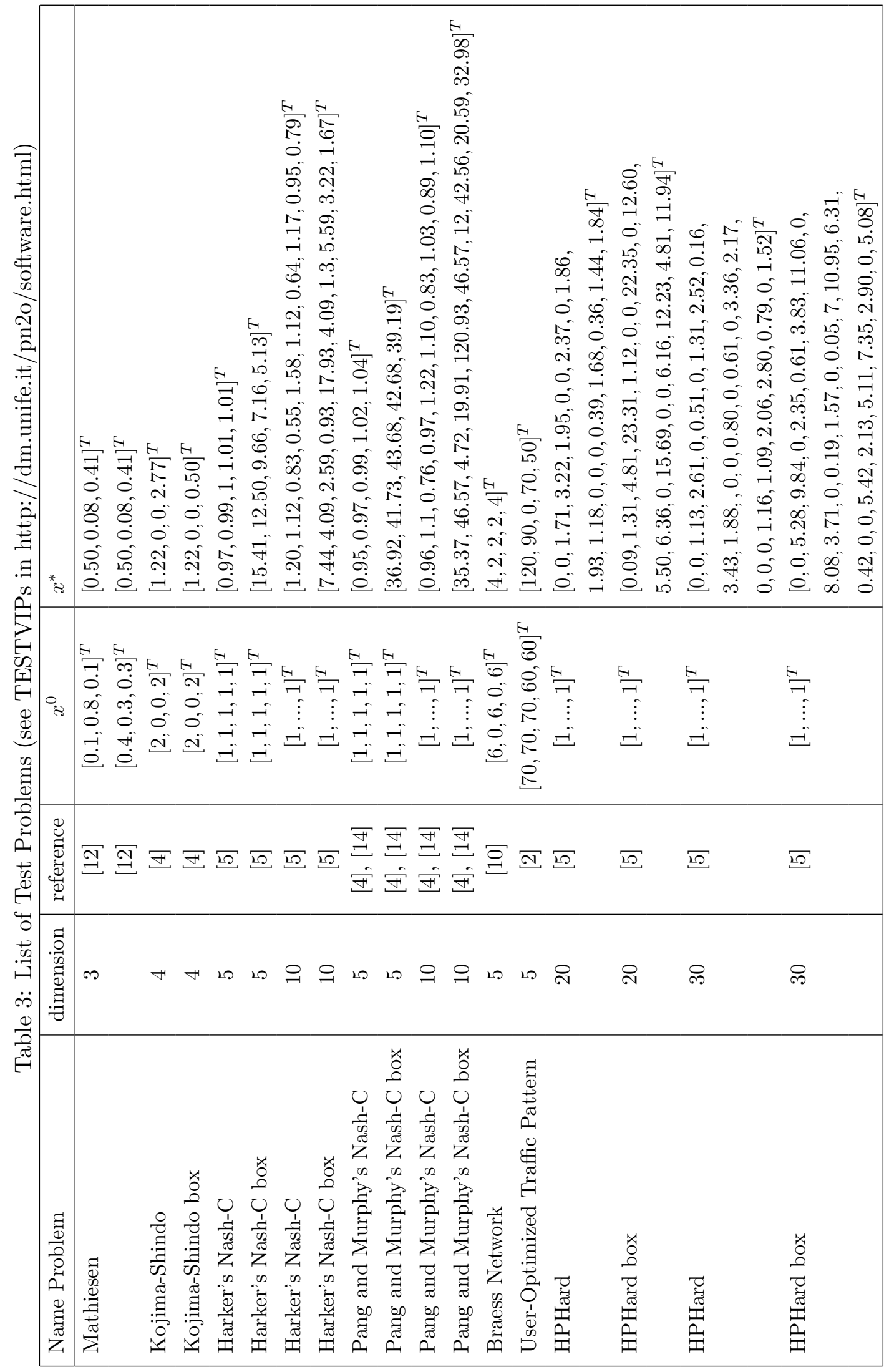




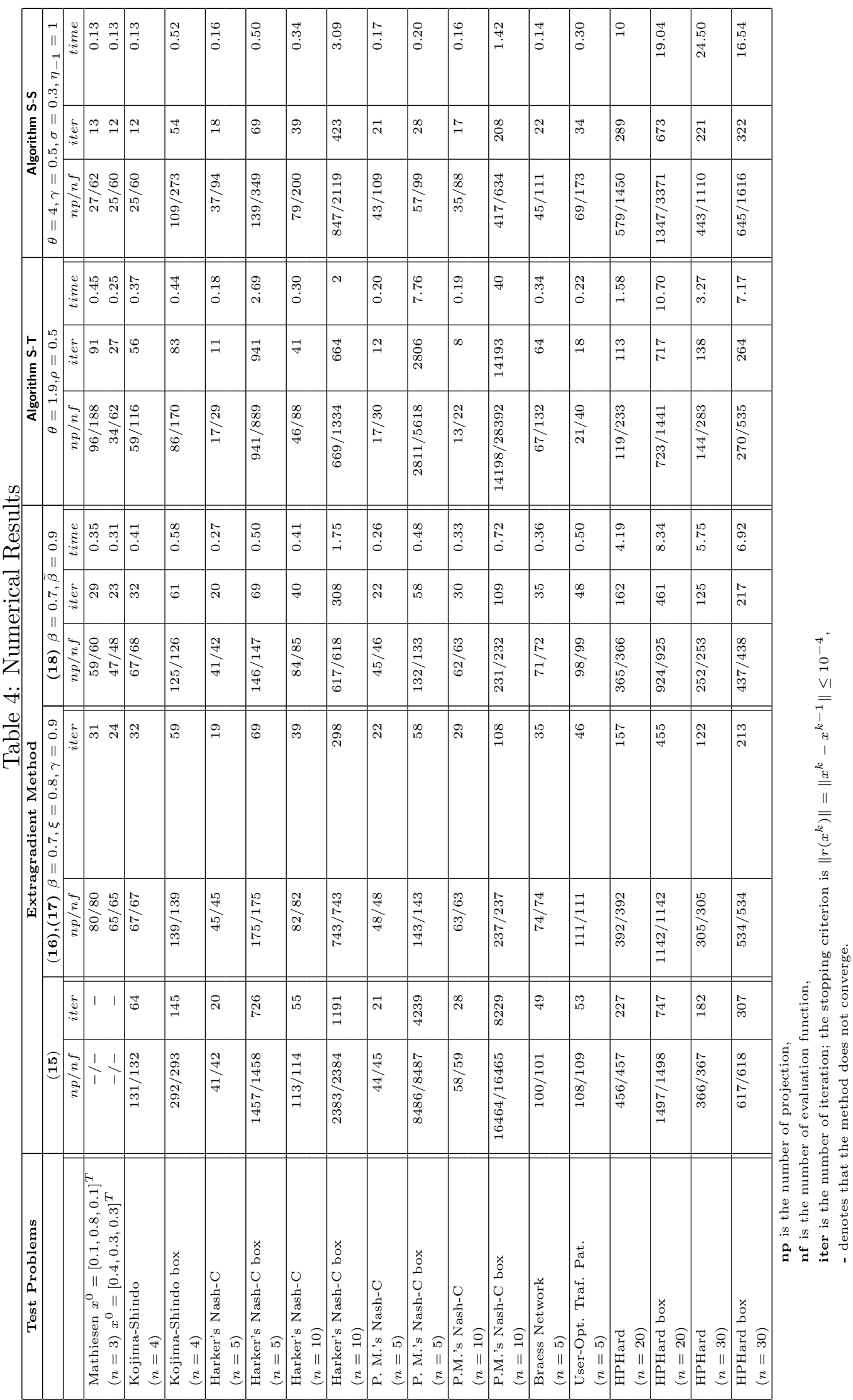




\section{Conclusion}

In this paper we reported a numerical analysis of the behavior of a set of extragradient-type methods that enable us to solve pseudomonotone VIPs and NCPs. In particular, we devised a convenient variant of the Khobotov's extragradient method that appears numerically effective above all for NCPs where one projection on the nonnegative orthant is very simple.

We compared other two extragradient-type methods: the first proposed by Solodov and Tseng can be very convenient for monotone VIPs while the second proposed by Solodov and Svaiter and called hyperplane projection method can be solve also pseudomonotone VIPs. This method appears very effective when the addition of a linear inequality constraint to the original feasible region does not increase too much the computational complexity of the special projections required by the scheme.

All the numerical results are reproducible by the codes available on the web site URL(http://dm.unife.it/pn2o/software.html).

This work is in progress, since we intend to update in the site by adding new significant test problems and by collecting further numerical results on the considered schemes and on the new schemes in the framework of extragradient-type methods.

\section{References}

[1] D.P. Bertsekas, J.N. Tsitsiklis Parallel and Distribuited Computation, Numerical Method, Prentice-Hall, London 1989.

[2] S. Dafermos Traffic equilibrium and variational inequalities, Transportation Science, 14 (1980), pp.42-54.

[3] F. Facchinei, J.-S. Pang Finite-Dimensional Variational Inequalities and Complementarity Problems, Springer Series in Operation Research, Springer (2003).

[4] S.A. Gabriel, J.-S. Pang NE/SQP: A robust algorithm for the nonlinear complementary problem, Mathematical Programming, 60 (1993), pp. 295-337. 
[5] P.T. Harker Accelerating the convergence of the diagonalization and projection algorithms for finite-dimensional variational inequalities, Mathematical Programming 41, 1988 (29-59).

[6] A.N. Iusem An iterative algorithm for the variational inequality problem, Computational and Applied Mathematics ,Vol.13, N.2, 1994 (103114).

[7] A.N. Iusem, B.F. Svaiter A variant of Korpelevich's method for variational inequalities with a new search strategy, Optimization, Vol. 42, 1997 (309-321).

[8] E.N. Khobotov Modification of the extra-gradient method for solving variational inequalities certain optimization problems, U.S.S.R. Computational Mathematics and Matematical Physics, 27 (1987), pp. 120127.

[9] G.M. Korpelevich The extragradient method for finding saddle points and other problems, Matekon, 13 (1977), pp. 35-49.

[10] P. Marcotte Application of Khobotov's algorithm to variational inequalities and network equilibrium problems, INFORM, 29 (1991).

[11] P. Marcotte, J.H. Wu On the convergence of projection methods application to the decomposition of affine variational inqualities, Journal of Optimization Theory and Applications, Vol.85, N.2, 1995 (347-362).

[12] L. Mathiesen An algorithm based on a sequence of linear complementary problems applied to a Walrasian ewuilibrium model: an example, Mathematical Programming, 37 1987, (1-18).

[13] The Math Works Inc. MatLab Optimization Toolbox v.2.0, User's Guide, 2002.

[14] F.H. Murphy, H.D. Sherali, A.L. Soyster A mathematical programming approach for determining oligopolistic market equilibrium, Mathematical Programming, 24 (1982), pp.92-106. 
[15] M. Patriksson Nonlinear Programming and Variational Inequality Problems, A Unified Approach, Applied Optimization, Vol.23, Kluwer Academic Pubblishers 1999.

[16] M. Sibony Méthodes itératives pour les équations et inéquations aux dérivés partielles nonlinéares de type monotone Calcolo, N.7, 1970, (65183).

[17] M.V. Solodov, P. Tseng Modifield projection type methods for monotone variational inequalities SIAM Journal Control Optimization, Vol.34, N.5, 1996 (1914-1830).

[18] M.V. Solodov, B.F. Svaiter A new projection method for variational inequality problems, SIAM Journal Control Optimization, Vol.37, N.3, 1999 (756-776).

[19] P. Tseng On linear convergence of iterative methods for variational inequality problem, Journal of Computational and Applied Mathematics 60, 1995 (237-252). 